Research Article

\title{
Breather Wave Solutions and Interaction Solutions for Two Mixed Calogero-Bogoyavlenskii-Schiff and Bogoyavlensky- Konopelchenko Equations
}

\author{
Hongcai Ma $\mathbb{D}^{1,2}$ Caoyin Zhang, ${ }^{1}$ and Aiping Deng ${ }^{1,2}$ \\ ${ }^{1}$ Department of Applied Mathematics, Donghua University, Shanghai 201620, China \\ ${ }^{2}$ Institute for Nonlinear Sciences, Donghua University, Shanghai 201620, China
}

Correspondence should be addressed to Hongcai Ma; hongcaima@hotmail.com

Received 10 March 2020; Revised 12 May 2020; Accepted 20 May 2020; Published 16 June 2020

Academic Editor: Antonio Scarfone

Copyright (C) 2020 Hongcai Ma et al. This is an open access article distributed under the Creative Commons Attribution License, which permits unrestricted use, distribution, and reproduction in any medium, provided the original work is properly cited.

\begin{abstract}
In this paper, based on a bilinear differential equation, we study the breather wave solutions by employing the extended homoclinic test method. By constructing the different forms, we also consider the interaction solutions. Furthermore, it is natural to analyse dynamic behaviors of three-dimensional plots.
\end{abstract}

\section{Introduction}

Recently, great attention has been paid to the study about exact solutions of nonlinear partial differential equations. So, it becomes more important to seek exact solutions of nonlinear partial differential equations (NLPDEs), which occur in many fields, such as chemistry, biology, optics, classical mechanics, acoustics, engineering, and social sciences. At present, many mathematicians have proposed a large number of methods to seek exact solutions, such as Bäcklund transformation [1], Hirota bilinear methods [2], homoclinic breather limit approach [3, 4], and Darboux transformation [5-12]. Among these methods, the Hirota bilinear method is one of the most critical and powerful methods. Recently, some new exact solutions of nonlinear partial differential equations have been constructed [13-22] by means of bilinear operator theories, so it has become an important research direction to study the dynamic properties of these new equations. In this article, the breather wave solutions will be discussed. On the basis of lump solution [23], the interaction solutions will be obtained.
The two mixed Calogero-Bogoyavlenskii-Schiff (CBS) and Bogoyavlensky-Konopelchenko (BK) equations [23] are usually written as

$$
\begin{gathered}
u_{t}+u_{2 x, y}+3 u_{x} u_{y}+\delta_{1} u_{y}+\delta_{2} w_{2 y}+\delta_{3} u_{x}+\delta_{4}\left(3 u_{x}^{2}+u_{3 x}\right) \\
+\delta_{5}\left(3 w_{2 y}^{2}+w_{4 y}\right)+\delta_{6}\left(3 u_{y} w_{2 y}+u_{3 y}\right)=0,
\end{gathered}
$$

where $u_{x}=w$ and $\delta_{i}, i=1, \cdots, 6$, are arbitrary constants. When the constants satisfy $\delta_{3}=\delta_{4}=\delta_{5}=\delta_{6}=0$, and $\delta_{5}=\delta_{6}=0$, the Calogero-Bogoyavlenskii-Schiff (CBS) and BogoyavlenskyKonopelchenko (CBS-BK) equations will become a generalized Calogero-Bogoyavlenskill-Schiff (gCBS) equation [24] and a generalized Calagero-Bogoyavlenskii Konopelchenko equation [25], respectively. The CBS equation was first constructed by Bogoyavlenskii and Schiff in different ways $[26,27]$. Namely, Bogoyavlenskii used the modified Lax formalism, whereas Schiff derived the same equation by reducing the self-dual Yang-Mills equation. In 2019, a class explicit lump solutions of the CBS-BK equation are 
constructed by using the Hirota bilinear approaches by Ren et al. [23]. The $(2+1)$-dimensional CBS equation also can be derived from the Korteweg-de Vries equation [28, 29]. Moreover, the BK equation is used as the interaction of a Rieman wave propagation [30], so we called the $(2+1)$-dimensional nonlinear partial differential equation (1) as gCBS-BK equation. These two equations have been widely studied in different ways [29, 31-40].

\section{The Bilinear Equation for gCBS-BK Equation}

If we take

$$
u=2 \partial_{x} \ln f
$$

where $f(x, y, t)$ is an unknown real function, the bilinear equation of Equation (1) can be presented

$$
\begin{array}{r}
{\left[D_{t} D_{x}+D_{x}^{3} D_{y}+\delta_{1} D_{x} D_{y}+\delta_{2} D_{y}^{2}+\delta_{3} D_{x}^{2}\right.} \\
\left.+\delta_{4} D_{x}^{4}+\delta_{5} D_{y}^{4}+\delta_{6} D_{x} D_{y}^{3}\right] f \cdot f=0,
\end{array}
$$

where $D_{t}, D_{x}$ are all bilinear derivative operators and $D$ -operator [2] is defined by

$D_{x}^{m} D_{t}^{n} a(x, t) \cdot b(x, t)=\left.\left(\partial_{t}-\partial_{t^{\prime}}\right)^{n}\left(\partial_{x}-\partial_{x}{ }^{\prime}\right)^{m} a(x, t) b\left(x^{\prime}, t^{\prime}\right)\right|_{x}{ }^{\prime}=x, t^{\prime}=t$,

where $m$ and $n$ are the positive integers, $a(x, t)$ is the function of $x$ and $t$, and $b(x, t)$ is the function of the formal variables $x^{\prime}$ and $t^{\prime}$.

\section{Breather Wave Solutions of CBS- BK Equation}

In this section, we will use the extended homoclinic text method $[41,42]$ to get the breather wave solutions of Equation (1). To start with,

$$
f(x, y, t)=k_{1} \exp \left(\xi_{1}\right)+\exp \left(-\xi_{1}\right)+k_{2} \cos \left(\xi_{2}\right)+a_{9},
$$

where $\xi_{1}$ and $\xi_{2}$ are defined by

$$
\left\{\begin{array}{l}
\xi_{1}=a_{1} x+a_{2} y+a_{3} t+a_{4}, \\
\xi_{2}=a_{5} x+a_{6} y+a_{7} t+a_{8}
\end{array}\right.
$$

where $a_{i}, i=1, \cdots, 8, k_{1}$, and $k_{2}$ are all real numbers. Substituting Equation (5) into Equation (3), we can get the following.where $a_{1}, a_{5}$, and $a_{7}$ are some free real numbers.

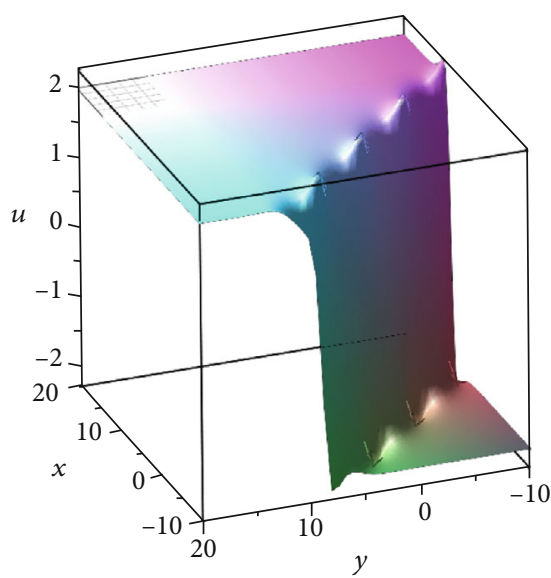

FIgURE 1: Spatiotemporal structure of solution (8) with the parameter selections $a_{1}=1, a_{2}=1, a_{3}=1, a_{6}=1, k_{1}=1, \delta_{1}=1, \delta_{2}$ $=1, \delta_{5}=1$, and $\delta_{6}=1$.

Case 1.

$a_{5}=0$,

$a_{7}=-\frac{a_{6}\left(3 a_{1} a_{2}^{2} \delta_{6}-a_{1} a_{6}^{2} \delta_{6}+4 a_{2}^{3} \delta_{5}-4 a_{2} a_{6}^{2} \delta_{5}+a_{1}^{3}+a_{1} \delta_{1}+2 a_{2} \delta_{2}\right)}{a_{1}}$ $a_{9}=0$.

Substituting Equation (7) into Equation (5), through the transformation (2), we have

$u(x, y, t)=\frac{2 k_{1} a_{1} \exp \left(\xi_{1}\right)-2 k_{1} a_{1} \exp \left(-\xi_{1}\right)-2 k_{2} a_{5} \sin \left(\xi_{2}\right)}{k_{1} \exp \left(\xi_{1}\right)+\exp \left(-\xi_{1}\right)+k_{2} \cos \left(\xi_{2}\right)}$,

where $\xi_{1}$ and $\xi_{2}$ are given by

$\left\{\begin{array}{l}\xi_{1}=a_{1} x+a_{2} y+a_{3} t+a_{4}, \\ \xi_{2}=a_{6} y-\frac{a_{6}\left(3 a_{1} a_{2}^{2} \delta_{6}-a_{1} a_{6}^{2} \delta_{6}+4 a_{2}^{3} \delta_{5}-4 a_{2} a_{6}^{2} \delta_{5}+a_{1}^{3}+a_{1} \delta_{1}+2 a_{2} \delta_{2}\right)}{a_{1}} t+a_{8},\end{array}\right.$

where $a_{i}, i=1, \cdots, 8, \delta_{1}, \delta_{2}, \delta_{5}$, and $\delta_{6}$ are real numbers. Figure 1 described the evolution of solution (8).

Case 2.

$$
\begin{aligned}
& a_{2}=0, \\
& a_{3}=\frac{a_{1} a_{7}}{a_{5}}, \\
& a_{6}=0, \\
& a_{9}=0,
\end{aligned}
$$

Substituting Equation (10) into Equation (5), through the transformation (2), we have 


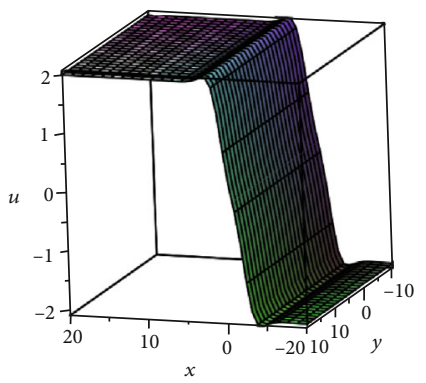

FIGURE 2: Spatiotemporal structure of solution (11) with the parameter selections $a_{1}=1, a_{5}=1$, and $a_{7}=1$.

$u(x, y, t)=\frac{2 k_{1} a_{1} \exp \left(\xi_{1}\right)-2 k_{1} a_{1} \exp \left(-\xi_{1}\right)-2 k_{2} a_{5} \sin \left(\xi_{2}\right)}{k_{1} \exp \left(\xi_{1}\right)+\exp \left(-\xi_{1}\right)+k_{2} \cos \left(\xi_{2}\right)}$,

where $\xi_{1}$ and $\xi_{2}$ are determined by

$$
\left\{\begin{array}{l}
\xi_{1}=a_{1} x+\frac{a_{1} a_{7}}{a_{5}} t+a_{4}, \\
\xi_{2}=a_{5} x+a_{7} t+a_{8}
\end{array}\right.
$$

where $a_{1}, a_{4}, a_{5}, a_{7}$, and $a_{8}$ are real numbers. Therefore, the dynamic behavior can be performed in Figure 2 .

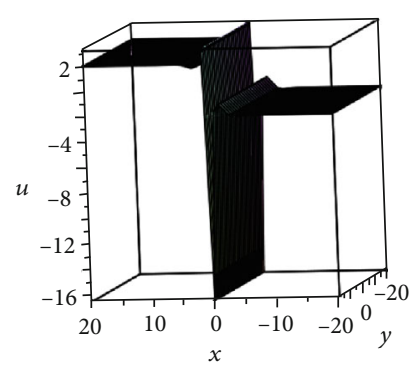

FIGURE 3: Spatiotemporal structure of solution (14) with the parameter selections $a_{1}=1, a_{5}=1, a_{7}=1, k_{1}=1, k_{2}=1$, and $\delta_{4}=1$.

Case 3.

$$
\begin{aligned}
& a_{2}=0, \\
& a_{3}=\frac{a_{1}\left(2 a_{1}^{2} a_{5} \delta_{4}+2 a_{5}^{3} \delta_{4}+a_{7}\right)}{a_{5}}, \\
& a_{6}=0, \\
& a_{9}=0, \\
& k_{1}=-\frac{k_{2}^{2} a_{5}^{2}}{4 k_{1}^{2}} .
\end{aligned}
$$

Substituting Equation (13) into Equation (5), through the transformation (2), we have

$$
u(x, y, t)=\frac{-\left(2 k_{2}^{2} a_{5}^{2} / 4 k_{1}^{2}\right) a_{1} \exp \left(\xi_{1}\right)+\left(2 k_{2}^{2} a_{5}^{2} / 4 k_{1}^{2}\right) a_{1} \exp \left(-\xi_{1}\right)-2 k_{2} a_{5} \sin \left(\xi_{2}\right)}{k_{1} \exp \left(\xi_{1}\right)+\exp \left(-\xi_{1}\right)+k_{2} \cos \left(\xi_{2}\right)}
$$

where $\xi_{1}$ and $\xi_{2}$ are given by

$$
\left\{\begin{array}{l}
\xi_{1}=a_{1} x+\frac{a_{1}\left(2 a_{1}^{2} a_{5} \delta_{4}+2 a_{5}^{3} \delta_{4}+a_{7}\right)}{a_{5}} t+a_{4} \\
\xi_{2}=a_{5} x+a_{7} t+a_{8}
\end{array}\right.
$$

where $a_{1}, a_{4}, a_{5}, a_{7}, a_{8}, k_{1}, k_{2}$, and $\delta_{4}$ are free real numbers. Figure 3 described the evolution of solution (14).
Case 4. Substituting $a_{1}, k_{1}$, and $a_{7}$ into Equation (5), through the transformation (2), we have

$$
u(x, y, t)=\frac{2 k_{1} a_{1} \exp \left(\xi_{1}\right)-2 k_{1} a_{1} \exp \left(-\xi_{1}\right)-2 k_{2} a_{5} \sin \left(\xi_{2}\right)}{k_{1} \exp \left(\xi_{1}\right)+\exp \left(-\xi_{1}\right)+k_{2} \cos \left(\xi_{2}\right)} .
$$

The evolution of solution (16) is described in Figure 4. $\xi_{1}$ and $\xi_{2}$ are given by

$$
\left\{\begin{array}{l}
\xi_{1}=-\frac{a_{2} a_{5}}{a_{6}} x+a_{2} y+a_{3} t+a_{4} \\
\xi_{2}=a_{5} x+a_{6} y+\frac{8 a_{2}^{2} a_{6}^{4} \delta_{5}-8 a_{6}^{6} \delta_{5}+4 a_{2}^{2} a_{5}^{3} a_{6}+4 a_{5}^{3} a_{6}^{3}-3 a_{2}^{2} a_{5}^{2} \delta_{3}-3 a_{2}^{3} a_{5}^{3} a_{6} \delta_{1}-3 a_{2}^{2} a_{6}^{2} \delta_{2}-3 a_{5}^{2} a_{6}^{2} \delta_{3}-3 a_{5} a_{6}^{3} \delta_{1}+a_{6}^{4} \delta_{2}}{3 a_{5}\left(a_{2}^{2}+a_{6}^{2}\right)} t+a_{8}
\end{array}\right.
$$

where $a_{2}, a_{3}, a_{4}, a_{5}, a_{6}, a_{8}, \delta_{1}$, and $\delta_{2}$ are real numbers. 


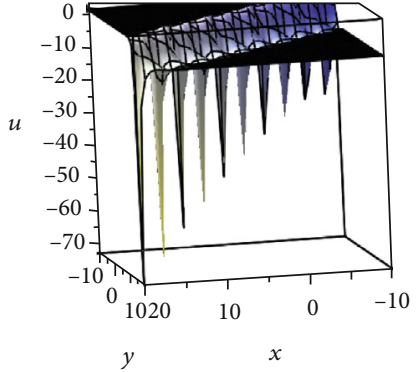

FIgURE 4: Spatiotemporal structure of solution (16) with the parameter selections $a_{2}=1, a_{5}=1, a_{6}=1, k_{2}=1, \delta_{1}=1, \delta_{2}=1$, $\delta_{3}=1$, and $\delta_{5}=1$.

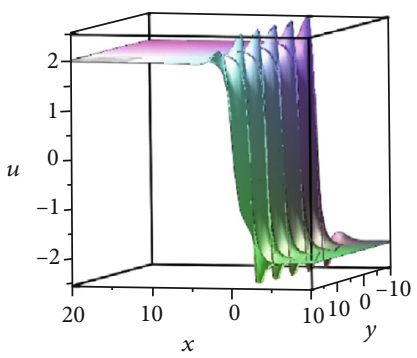

FIGURE 5: Spatiotemporal structure of solution (19) $a_{1}=1, a_{5}=1$, $a_{6}=1, k_{1}=1, k_{2}=1, \delta_{3}=1$, and $\delta_{6}=1$.

Case 5.

$$
\begin{aligned}
& a_{2}=0, \\
& a_{3}=\frac{a_{1}\left(a_{1}^{2} a_{6}-2 a_{5} \delta_{3}\right)}{2 a_{5}}, \\
& a_{7}=a_{6}^{3} \delta_{6}+\frac{a_{1}^{2} a_{6}}{2}+a_{5}^{2} a_{6}-a_{5} \delta_{3}-a_{6} \delta_{1} .
\end{aligned}
$$

Substituting Equation (18) into Equation (5), through the transformation (2), we have

$u(x, y, t)=\frac{2 k_{1} a_{1} \exp \left(\xi_{1}\right)-2 k_{1} a_{1} \exp \left(-\xi_{1}\right)-2 k_{2} a_{5} \sin \left(\xi_{2}\right)}{k_{1} \exp \left(\xi_{1}\right)+\exp \left(-\xi_{1}\right)+k_{2} \cos \left(\xi_{2}\right)}$.

The evolution of solution (19) is described in Figure 5.

$\xi_{1}$ and $\xi_{2}$ are given by

$$
\left\{\begin{array}{l}
\xi_{1}=a_{1} x+\frac{a_{1}\left(a_{1}^{2} a_{6}-2 a_{5} \delta_{3}\right)}{2 a_{5}} t+a_{4}, \\
\xi_{2}=a_{5} x+a_{6} y+\left(a_{6}^{3} \delta_{6}+\frac{a_{1}^{2} a_{6}}{2}+a_{5}^{2} a_{6}-a_{5} \delta_{3}-a_{6} \delta_{1}\right) t+a_{8},
\end{array}\right.
$$

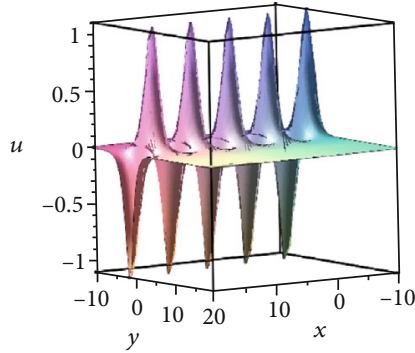

Figure 6: Spatiotemporal structure of solution (22) with the parameter selections $a_{2}=1, a_{5}=1, a_{7}=1, k_{1}=1, k_{2}=1, \delta_{1}=1$, $\delta_{5}=1$, and $\delta_{6}=1$.

where $a_{1}, a_{4}, a_{5}, a_{6}, a_{8}, k_{1}, k_{2}, \delta_{3}$, and $\delta_{6}$ are free real numbers. The three-dimensional dynamic figure can be drawn as Figure 5.

Case 6.

$$
\begin{aligned}
& a_{1}=0, \\
& a_{3}=-a_{2}\left(a_{2}^{2} \delta_{6}-a_{5}^{2}+\delta_{1}\right), \\
& a_{6}=0 .
\end{aligned}
$$

Substituting Equation (21) into Equation (5), through the transformation (2), we have

$$
u(x, y, t)=\frac{2 k_{1} a_{1} \exp \left(\xi_{1}\right)-2 k_{1} a_{1} \exp \left(-\xi_{1}\right)-2 k_{2} a_{5} \sin \left(\xi_{2}\right)}{k_{1} \exp \left(\xi_{1}\right)+\exp \left(-\xi_{1}\right)+k_{2} \cos \left(\xi_{2}\right)},
$$

where $\xi_{1}$ and $\xi_{2}$ are defined by

$$
\left\{\begin{array}{l}
\xi_{1}=a_{2} y-a_{2}\left(a_{2}^{2} \delta_{6}-a_{5}^{2}+\delta_{1}\right) t+a_{4} \\
\xi_{2}=a_{5} x+a_{7} t+a_{8}
\end{array}\right.
$$

where $a_{2}, a_{4}, a_{5}, a_{7}, a_{8}, k_{1}, k_{2}, \delta_{1}, \delta_{5}$, and $\delta_{6}$ are some free real numbers. The figure is given as Figure 6.

\section{Case 7.}

$$
\begin{aligned}
& a_{2}=-a_{6}, \\
& a_{3}=\frac{4 a_{1}^{3} a_{6}-3 a_{1}^{2} \delta_{3}+3 a_{1} a_{6} \delta_{1}-a_{6}^{2} \delta_{2}}{3 a_{1}}, \\
& a_{7}=\frac{4 a_{1}^{3} a_{6}-3 a_{1}^{2} \delta_{3}-3 a_{1} a_{6} \delta_{1}-a_{6}^{2} \delta_{1}}{3 a_{1}}, \\
& k_{1}=\frac{k_{2}^{2}}{4},
\end{aligned}
$$




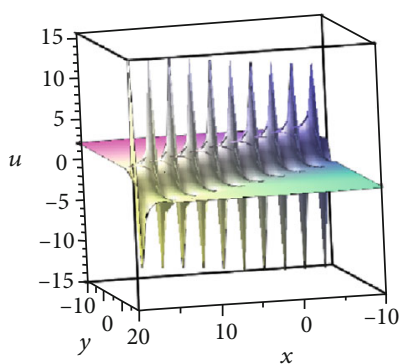

FIGURE 7: Spatiotemporal structure of solution (25) with the parameter selections $a_{1}=1, a_{6}=1, a_{7}=1, k_{2}=1, \delta_{1}=1, \delta_{2}=1$, $\delta_{3}=1$, and $\delta_{5}=1$.

where $a_{1}, a_{6}, a_{7}, k_{2}, \delta_{1}, \delta_{2}, \delta_{3}$, and $\delta_{5}$ are free real numbers. Substituting Equation (24) into Equation (5), through the transformation (2), we have

$u(x, y, t)=\frac{\left(2 k_{2}^{2} / 4\right) a_{1} \exp \left(\xi_{1}\right)-\left(2 k_{2}^{2} / 4\right) a_{1} \exp \left(-\xi_{1}\right)-2 k_{2} a_{5} \sin \left(\xi_{2}\right)}{\left(k_{2}^{2} / 4\right) \exp \left(\xi_{1}\right)+\exp \left(-\xi_{1}\right)+k_{2} \cos \left(\xi_{2}\right)}$.

The figure is given as Figure 7.

$\xi_{1}$ and $\xi_{2}$ are followed by

$$
\left\{\begin{array}{l}
\xi_{1}=a_{1} x+a_{2} y-\frac{4 a_{1}^{3} a_{6}+3 a_{1}^{2} \delta_{3}+3 a_{1} a_{6} \delta_{1}+a_{6}^{2} \delta_{2}}{3 a_{6}^{2} \delta_{1}} t+a_{4}, \\
\xi_{2}=-a_{1} x+a_{6} y+\frac{4 a_{1}^{3} a_{6}+3 a_{1}^{2} \delta_{3}-3 a_{1} a_{6} \delta_{1}+a_{6}^{2} \delta_{1}}{3 a_{1}} t+a_{8}
\end{array}\right.
$$

where $a_{1}, a_{4}, a_{5}, a_{6}, a_{7}, a_{8}, k_{2}, \delta_{1}, \delta_{2}, \delta_{3}$, and $\delta_{5}$ are free real numbers.

Case 8.

$$
\begin{aligned}
& a_{3}=-\frac{4 a_{1}^{3} a_{6}+3 a_{1}^{2} \delta_{3}+3 a_{1} a_{6} \delta_{1}+a_{6}^{2} \delta_{2}}{3 a_{6}^{2} \delta_{1}}, \\
& a_{5}=-a_{1}, \\
& a_{7}=\frac{4 a_{1}^{3} a_{6}+3 a_{1}^{2} \delta_{3}-3 a_{1} a_{6} \delta_{1}+a_{6}^{2} \delta_{1}}{3 a_{1}},
\end{aligned}
$$

where $a_{1}, a_{6}, a_{7}, k_{2}, \delta_{1}, \delta_{2}, \delta_{3}$, and $\delta_{5}$ are free real numbers. Substituting Equation (27) into Equation (5), through the transformation (2), we have

$u(x, y, t)=\frac{2 k_{1} a_{1} \exp \left(\xi_{1}\right)-2 k_{1} a_{1} \exp \left(-\xi_{1}\right)-2 k_{2} a_{5} \sin \left(\xi_{2}\right)}{k_{1} \exp \left(\xi_{1}\right)+\exp \left(-\xi_{1}\right)+k_{2} \cos \left(\xi_{2}\right)}$.

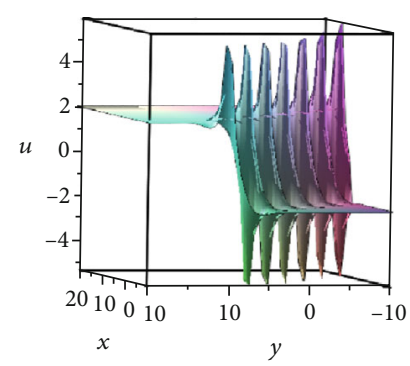

Figure 8: Spatiotemporal structure of solution (28) with the parameter selections $a_{1}=1, a_{6}=1, a_{7}=1, k_{2}=1, \delta_{1}=1, \delta_{2}=1$, $\delta_{3}=1$, and $\delta_{5}=1$.

The figure is drawn as Figure 8 .

$\xi_{1}$ and $\xi_{2}$ are defined by

$$
\left\{\begin{array}{l}
\xi_{1}=a_{1} x+a_{2} y-\frac{4 a_{1}^{3} a_{6}+3 a_{1}^{2} \delta_{3}+3 a_{1} a_{6} \delta_{1}+a_{6}^{2} \delta_{2}}{3 a_{6}^{2} \delta_{1}} t+a_{4}, \\
\xi_{2}=-a_{1} x+a_{6} y+\frac{4 a_{1}^{3} a_{6}+3 a_{1}^{2} \delta_{3}-3 a_{1} a_{6} \delta_{1}+a_{6}^{2} \delta_{1}}{3 a_{1}} t+a_{8},
\end{array}\right.
$$

where $a_{1}, a_{2}, a_{4}, a_{6}, a_{7}, a_{8}, k_{2}, \delta_{1}, \delta_{2}, \delta_{3}$, and $\delta_{5}$ are free real numbers.

\section{Interaction Solutions of CBS-BK System}

4.1. Interaction between a Lump and One-Kink Soliton. With the help of Maple, we will discuss the interaction between a lump and one-kink soliton by taking $f(x, y, t)$ as a combination of positive quadratic function and one exponential function, that is,

$$
f(x, y, t)=\xi_{1}^{2}+\xi_{2}^{2}+\exp \left(\xi_{3}\right)+a_{9}
$$

where $\xi_{1}, \xi_{2}$, and $\xi_{3}$ are defined by

$$
\left\{\begin{array}{l}
\xi_{1}=a_{1} x+a_{2} y+a_{3} t+a_{4} \\
\xi_{2}=a_{5} x+a_{6} y+a_{7} t+a_{8} \\
\xi_{3}=k_{1} x+p_{1} y+q_{1} t+r_{1}
\end{array}\right.
$$

where $a_{i}, i=1, \cdots, 9, p_{1}, k_{1}, r_{1}$, and $q_{1}$ are all real numbers. In order to get the interaction solutions of Equation (1), substituting Equation (30) into Equation (2), 


$$
u(x, y, t)=\frac{4 a_{1} \xi_{1}+4 a_{2} \xi_{2}+2 k_{1} \exp \left(\xi_{3}\right)}{\xi_{1}^{2}+\xi_{2}^{2}+\exp \left(\xi_{3}\right)+a_{9}}
$$

where $\xi_{1}, \xi_{2}$, and $\xi_{3}$ are defined by

$$
\left\{\begin{array}{l}
\xi_{1}=a_{1} x+a_{2} y+a_{3} t+a_{4}, \\
\xi_{2}=a_{5} x+a_{6} y+a_{7} t+a_{8}, \\
\xi_{3}=k_{1} x+p_{1} y+q_{1} t+r_{1},
\end{array}\right.
$$

where $a_{i}, i=1, \cdots, 9, p_{1}, k_{1}, r_{1}$, and $q_{1}$ are all real numbers. Substituting Equation (30) into Equation (3), through complex analysis and calculations, we can have the following.
Case 1.

$$
\begin{aligned}
& a_{1}=0, \\
& a_{6}=0, \\
& k_{1}=0, \\
& \delta_{2}=0, \\
& \delta_{4}=0, \\
& \delta_{5}=0, \\
& q_{1}=\frac{p_{1}\left(a_{2} \delta_{6} p_{1}^{2}-a_{3}\right)}{a_{2}}, \\
& \delta_{1}=-\frac{a_{7}}{a_{5}},
\end{aligned}
$$

where $a_{2}, a_{3}, a_{5}, a_{7}, p_{1}$, and $\delta_{6}$ are free real numbers. Substituting Equation (34) into Equation (32), we have

$$
u(x, y, t)=\frac{2 a_{5}\left(a_{5} x+a_{6} y+a_{7} t+a_{8}\right)}{a_{9}+\left(a_{2} y+a_{3} t+a_{4}\right)^{2}+\left(a_{5} x+a_{7} t+a_{8}\right)^{2}+b_{1} \exp \left(p_{1} y+\left(p_{1}\left(a_{2} \delta_{6} p_{1}^{2}-a_{3}\right) / a_{2}\right) t+r_{1}\right)} .
$$

Case 2.

$$
\begin{aligned}
& a_{1}=0, \\
& a_{5}=0, \\
& \delta_{2}=0, \\
& \delta_{5}=0, \\
& \delta_{6}=0, \\
& a_{3}=\frac{a_{7} a_{2}}{a_{6}}, \\
& \delta_{1}=\frac{a_{6} k_{1}^{2}+a_{7}}{a_{6}}, \\
& q_{1}=-\frac{a_{6} k_{1}^{3} \delta_{4}+a_{6} k_{1} \delta_{3}-a_{7} p_{1}}{a_{6}},
\end{aligned}
$$

where $a_{2}, a_{6}, a_{7}, k_{1}, p_{1}, \delta_{3}$, and $\delta_{4}$ are some free real numbers. Substituting Equation (36) into Equation (32), we have

$$
u(x, y, t)=\frac{4 a_{1} \xi_{1}+4 a_{2} \xi_{2}+\exp \left(\xi_{3}\right)}{\xi_{1}^{2}+\xi_{2}^{2}+\exp \left(\xi_{3}\right)+a_{9}}
$$

where $\xi_{1}, \xi_{2}$, and $\xi_{3}$ are defined by

$$
\left\{\begin{array}{l}
\xi_{1}=a_{2} y+\frac{a_{7} a_{2}}{a_{6}} t+a_{4}, \\
\xi_{2}=a_{6} y+a_{7} t+a_{8}, \\
\xi_{3}=k_{1} x+p_{1} y-\frac{a_{6} k_{1}^{3} \delta_{4}+a_{6} k_{1} \delta_{3}-a_{7} p_{1}}{a_{6}} t+r_{1},
\end{array}\right.
$$

where $a_{2}, a_{4}, a_{6}, a_{7}, a_{8}, k_{1}, p_{1}, r_{1}, \delta_{3}$, and $\delta_{4}$ are some free real numbers.

In order to obtain the dynamic feature, we choose Case 2 to analyse. The three-dimensional dynamic graphs are drawn as Figure 9. We can find that the lump waves and the exponential function waves interact with each other and keep moving in the opposite direction.

4.2. Interaction between a Lump and Periodic Waves. In order to get interaction solutions between a lump and periodic waves, we will take $f$ as the combination of positive function and hyperbolic cosine function. Therefore, $f$ can be determined by

$$
f(x, y, t)=\xi_{1}^{2}+\xi_{2}^{2}+b_{1} \cos \left(\xi_{3}\right)+a_{9}
$$

where variables are defined by

$$
\left\{\begin{array}{l}
\xi_{1}=a_{1} x+a_{2} y+a_{3} t+a_{4}, \\
\xi_{2}=a_{5} x+a_{6} y+a_{7} t+a_{8}, \\
\xi_{3}=k_{1} x+p_{1} y+q_{1} t+r_{1},
\end{array}\right.
$$




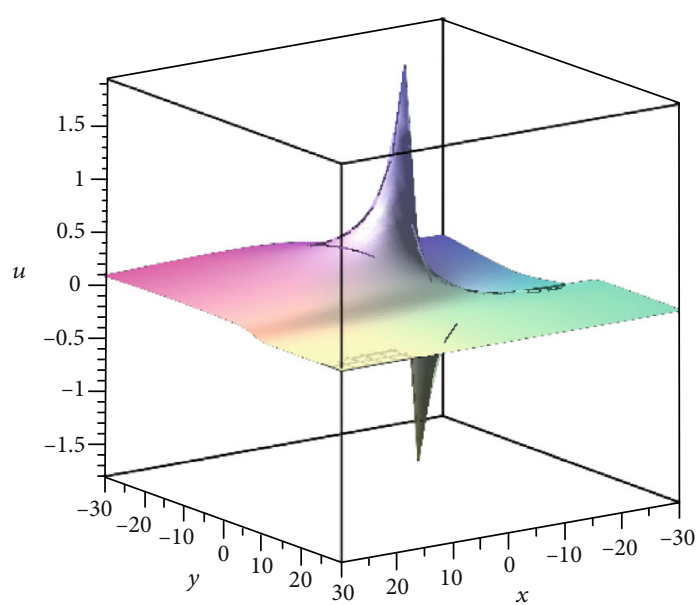

(a)

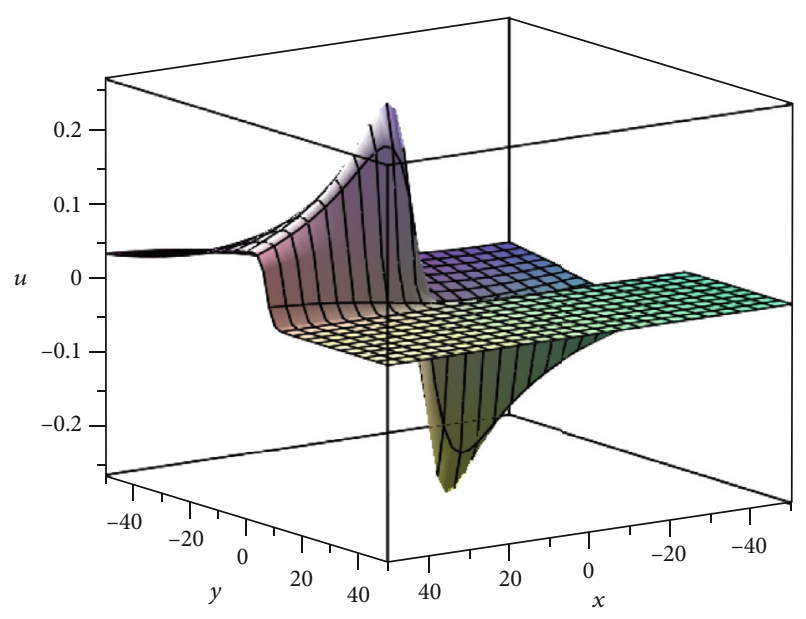

(b)

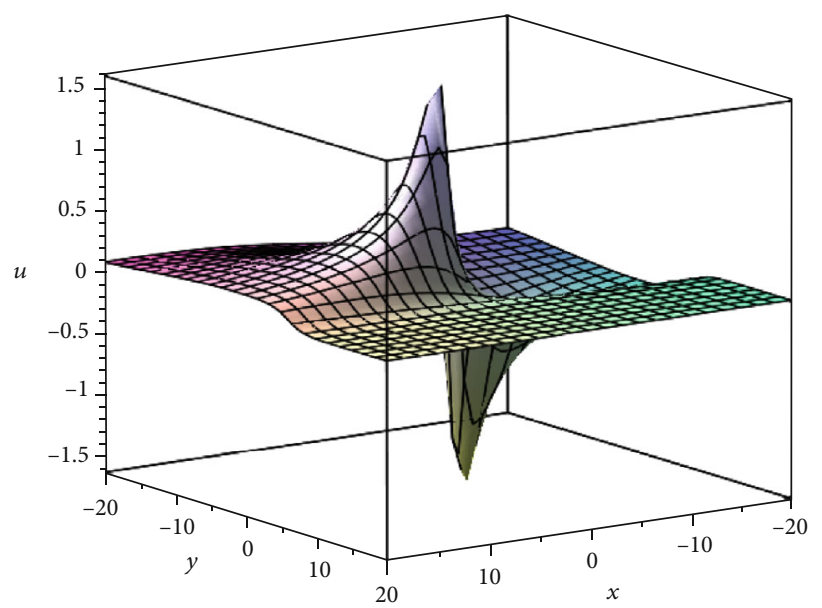

(c)

FiguRE 9: Spatiotemporal structure of solution (37) with the parameter selections: (a) $t=-10, a_{2}=1, p_{1}=1, a_{3}=1, a_{7}=1, a_{5}=1$, and $\delta_{6}=1$; (b) $t=0, a_{2}=1, p_{1}=1, a_{3}=1, a_{7}=1, a_{5}=1$, and $\delta_{6}=1$; (c) $t=-10, a_{2}=1, p_{1}=1, a_{3}=1, a_{7}=1, a_{5}=1$, and $\delta_{6}=1$.

where $a_{i}, i=1, \cdots, 9, p_{1}, k_{1}, r_{1}$, and $q_{1}$ are all real numbers. Substituting Equation (39) into Equation (2), we can get the interaction solutions of Equation (1):

$$
u(x, y, t)=\frac{4 a_{1} \xi_{1}+4 a_{2} \xi_{2}-2 b_{1} k_{1} \sin \left(\xi_{3}\right)}{\xi_{1}^{2}+\xi_{2}^{2}+b_{1} \cos \left(\xi_{3}\right)+a_{9}}
$$

where $\xi_{1}, \xi_{2}$, and $\xi_{3}$ are defined by

$$
\left\{\begin{array}{l}
\xi_{1}=a_{1} x+a_{2} y+a_{3} t+a_{4}, \\
\xi_{2}=a_{5} x+a_{6} y+a_{7} t+a_{8}, \\
\xi_{3}=k_{1} x+p_{1} y+q_{1} t+r_{1},
\end{array}\right.
$$

where $a_{i}, i=1, \cdots, 9, p_{1}, k_{1}, r_{1}$, and $q_{1}$ are real numbers. Through long and tedious calculations, we can get the following relations between the parameters.where $a_{5}, a_{7}, b_{1}, p_{1}, \delta_{1}$, $\delta_{2}$, and $\delta_{6}$ are free real numbers.where $a_{5}, a_{6}, a_{7}, p_{1}, b_{1}, \delta_{1}$, and $\delta_{6}$ are free real numbers.where $a_{2}, a_{3}, a_{5}, a_{7}, p_{1}$, and $\delta_{6}$ are some free real numbers.where $a_{5}, p_{1}, \delta_{1}$, and $\delta_{6}$ are free real numbers.

Case 1.

$$
\begin{aligned}
& a_{1}=0, \\
& a_{2}=0, \\
& a_{3}=0, \\
& a_{6}=0, \\
& q_{1}=p_{1}\left(P_{1}^{2} \delta_{6}-\delta_{1}\right), \\
& \delta_{3}=-\frac{a_{7}}{a_{5}}, \\
& \delta_{4}=-\frac{b_{1}^{2} p_{1}^{2} \delta_{2}}{a_{5}^{4}}, \\
& \delta_{5}=\frac{\delta_{2}}{p_{1}^{2}},
\end{aligned}
$$


Case 2.

$$
\begin{aligned}
& a_{1}=0, \\
& a_{2}=0, \\
& a_{3}=0, \\
& k_{1}=0, \\
& q_{1}=-p_{1} \delta_{1}, \\
& \delta_{2}=-\frac{a_{5} p_{1}^{2} \delta_{6}}{a_{6}}, \\
& \delta_{3}=\frac{a_{6} p_{1}^{2} \delta_{6}-2 a_{6} \delta_{1}-2 a_{7}}{a_{5}},
\end{aligned}
$$

Case 3.

$$
\begin{aligned}
& a_{1}=0, \\
& a_{6}=0, \\
& k_{1}=0, \\
& q_{1}=\frac{p_{1}\left(a_{2} p_{1}^{2} \delta_{6}+a_{3}\right)}{a_{2}}, \\
& \delta_{1}=-\frac{a_{3}}{a_{2}}, \\
& \delta_{2}=0, \\
& \delta_{3}=-\frac{a_{7}}{a_{5}}, \\
& \delta_{4}=0, \\
& \delta_{5}=0,
\end{aligned}
$$

Case 4.

$$
\begin{aligned}
& a_{2}=0, \\
& a_{3}=-a_{1} \delta_{3}, \\
& k_{1}=0, \\
& q_{1}=p_{1}^{3} \delta_{6}-p_{1} \delta_{1}, \\
& \delta_{2}=0, \\
& \delta_{4}=0, \\
& \delta_{5}=0,
\end{aligned}
$$

When we change the coefficients of the equation, the value of Equation (47) will be different accordingly. In order to obtain the dynamic feature, we choose Case 2 to analyse. Taking Equation (44) into Equation (41), we can get

$u(x, y, t)=\frac{2 a_{5}\left(a_{5}+a_{7} t+a_{8}\right)}{a_{9}+a_{4}^{2}+\left(a_{5} x+a_{6} y+a_{7} t+a_{8}\right)^{2}+b_{1} \cos \left(p_{1} y-p_{1} \delta_{1} t+r_{1}\right)}$.

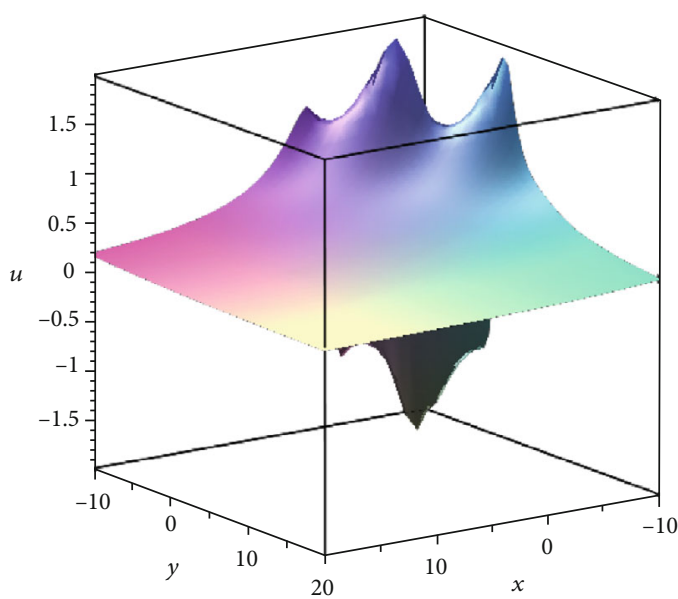

(a)

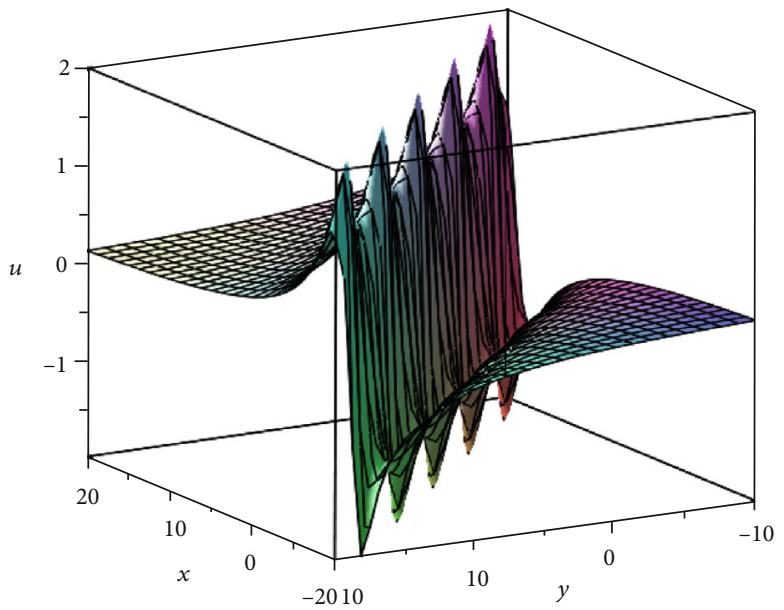

(b)

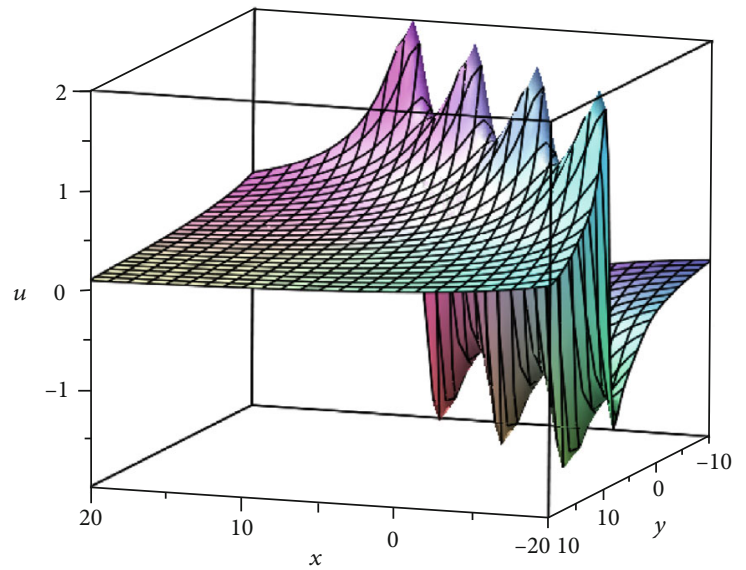

(c)

FIgURE 10: Spatiotemporal structure of solution (47) with the parameter selections: (a) $t=0, a_{5}=1, a_{6}=1, a_{7}=1, p_{1}=1, b_{1}=1$, $\delta_{1}=1$, and $\delta_{6}=1$; (b) $t=10, a_{5}=1, a_{6}=1, a_{7}=1, p_{1}=1, b_{1}=1, \delta_{1}=$ 1 , and $\delta_{6}=1 ;$ (c) $a_{5}=1, a_{6}=1, a_{7}=1, p_{1}=1, b_{1}=1, \delta_{1}=1$, and $\delta_{6}=1$.

With the help of Maple, the three-dimensional dynamic graphs are drawn as Figure 10. We can find that lump waves and periodic waves interact with each other and keep moving in the opposite direction. 


\section{Conclusions}

In this paper, based on a bilinear differential equation, we study the breather wave solutions and the interaction solutions of the mixed Calogero-Bogoyavlenskii-Schiff and Bogoyavlensky-Konopelchenko equations. Compared with the existing results in the literature, our results are new. It will be extensively used to report many attractive physical phenomena in the fields of acoustics, heat transfer, fluid dynamics, classical mechanics, and so on. It is demonstrated that the Hirota operators are very simple and powerful in constructing new nonlinear differential equations, which possess nice math properties. It is interesting to study the interaction solutions between soliton solutions and period solution by making $f$ as a combination of exponential function and trigonometry function. However, this method can be applied to those equations which have Hirota bilinear forms. Furthermore, we also can study the quardrilinear forms and even polylinearity forms of this equation in the future. These questions may also be interesting and worth studying.

\section{Data Availability}

No data were used to support this study.

\section{Conflicts of Interest}

The authors declare that they have no conflicts of interest.

\section{Acknowledgments}

The work is supported by the National Natural Science Foundation of China (project Nos. 11371086, 11671258, and 11975145), the Fund of Science and Technology Commission of Shanghai Municipality (project No. 13ZR1400100), the Fund of Donghua University, Institute for Nonlinear Sciences, and the Fundamental Research Funds for the Central Universities.

\section{References}

[1] C. Rogers and W. F. Shadwick, Backlund Transformations and Their Applications, Academic Press, New York, 1982.

[2] R. Hirota, The Direct Method in Soliton Theory, Cambridge University Press, Cambridge, United Kingdom, 2004.

[3] C. Y. Qin, S. F. Tian, X. B. Wang, and T. T. Zhang, "On breather waves, rogue waves and solitary waves to a generalized (2+1)-dimensional Camassa-Holm-Kadomtsev-Petviashvili equation," Communications in Nonlinear Science and Numerical Simulation, vol. 62, pp. 378-385, 2018.

[4] X.-w. Yan, S.-f. Tian, M.-j. Dong, L. Zhou, and T.-t. Zhang, "Characteristics of solitary wave, homoclinic breather wave and rogue wave solutions in a $(2+1)$-dimensional generalized breaking soliton equation," Computers \& Mathematics with Applications, vol. 76, no. 1, pp. 179-186, 2018.

[5] X. N. Gao, S. Y. Lou, and X. Y. Tang, "Bosonization, singularity analysis, nonlocal symmetry reductions and exact solutions of supersymmetric KdV equation," Journal of High Energy Physics, vol. 2013, no. 5, p. 29, 2013.
[6] B. Ren, "The (2+1)-dimensional Konopelchenko-Dubrovsky equation: nonlocal symmetries and interaction solutions," Nonlinear Dynamics, vol. 86, no. 3, pp. 1855-1862, 2016.

[7] B. Ren, "Symmetry reduction related with nonlocal symmetry for Gardner equation," Communications in Nonlinear Science and Numerical Simulation, vol. 42, pp. 456-463, 2017.

[8] Y. L. Dang, H. J. Li, and J. Lin, "Soliton solutions in nonlocal nonlinear coupler," Nonlinear Dynamics, vol. 88, no. 1, pp. 489-501, 2017.

[9] J. Lin, X. W. Jin, X. L. Gao, and S. Y. Lou, "Solitons on a periodic wave background of the modified KdV-Sine-Gordon equation," Communications in Theoretical Physics, vol. 70, no. 2, pp. 119-126, 2018.

[10] Y. Yıldırım and E. Yaşar, "Multiple exp-function method for soliton solutions of nonlinear evolution equations," Chinese Physics B, vol. 26, no. 7, p. 070201, 2017.

[11] A. R. Adem, Y. Yildirim, and E. Yaşar, "Soliton solutions to the non-local Boussinesq equation by multiple exp-function scheme and extended Kudryashov's approach," Pramana Journal of Physics, vol. 92, no. 2, pp. 1-11, 2019.

[12] Y. Yildäräm, E. Yasar, A. R. Adem, and E. Yasar, "A novel scheme for nonlinear evolution equations using symbolic computations," Journal of Applied Nonlinear Dynamics, vol. 8, no. 3, pp. 463-473, 2019.

[13] X. Lü, S.-T. Chen, and W.-X. Ma, "Constructing lump solutions to a generalized Kadomtsev-Petviashvili-Boussinesq equation," Nonlinear Dynamics, vol. 86, no. 1, pp. 523-534, 2016.

[14] W.-x. Ma, Y. Zhang, Y. Tang, and T. Junyi, "Hirota bilinear equations with linear subspaces of solutions," Applied Mathematics and Computation, vol. 218, no. 13, pp. 7174-7183, 2012.

[15] C.-G. Shi, B.-Z. Zhao, and W.-X. Ma, "Exact rational solutions to a Boussinesq-like equation in (1+1)-dimensions," Applied Mathematics Letters, vol. 48, pp. 170-176, 2015.

[16] Y. Zhang and W.-X. Ma, "Rational solutions to a KdV-like equation," Applied Mathematics and Computation, vol. 256, pp. 252-256, 2015.

[17] H.-C. Ma and A.-P. Deng, "Lump solution of (2+1)-dimensional Boussinesq equation," Communications in Theoretical Physics, vol. 65, no. 5, pp. 546-552, 2016.

[18] Y. Yıldırım and E. Yaşar, "An extended Korteweg-de Vries equation: multisoliton solutions and conservation laws," Nonlinear Dynamics, vol. 90, no. 3, pp. 15711579, 2017.

[19] E. Yaşar, Y. Yıldırım, and A. R. Adem, "Extended transformed rational function method to nonlinear evolution equations," International Journal of Nonlinear Sciences and Numerical Simulation, vol. 20, no. 6, pp. 691-701, 2019.

[20] Y. Yildırım and E. Yasar, "Wronskian solutions of $(2+1)$ dimensional nonlocal Ito equation," Communications Faculty Of Science University of Ankara Series A1Mathematics and Statistics, vol. 67, no. 2, pp. 126-138, 2018.

[21] A. R. Adem, Y. Yildirim, and E. Yașar, "Complexiton solutions and soliton solutions: (2+1)-dimensional Date-JimboKashiwara-Miwa equation," Pramana, vol. 92, no. 3, p. 36, 2019.

[22] Y. Yildirim, E. Yasar, and A. R. Adem, "A multiple expfunction method for the three model equations of shallow water waves," Nonlinear Dynamics, vol. 89, no. 3, pp. 22912297, 2017. 
[23] B. Ren, W. X. Ma, and J. Yu, "Lump solutions for two mixed Calogero-Bogoyavlenskii-Schiff and BogoyavlenskyKonopelchenko equations," Communications in Theoretical Physics, vol. 71, no. 6, pp. 658-662, 2019.

[24] S.-T. Chen and W.-X. Ma, "Lump solutions of a generalized Calogero-Bogoyavlenskii-Schiff equation," Computers \& Mathematics with Applications, vol. 76, no. 7, pp. 1680-1685, 2018.

[25] S.-T. Chen and W.-X. Ma, "Lump solutions to a generalized Bogoyavlensky-Konopelchenko equation," Frontiers of Mathematics in China, vol. 13, no. 3, pp. 525-534, 2018.

[26] O. I. Bogoyavlenskii, "Overturning solitons in new twodimensional integrable equations," Mathematics of the USSRIzvestiya, vol. 34, no. 2, pp. 245-259, 1990.

[27] J. Schiff, "Integrability of Chern-Simons-Higgs vortex equations and a reduction of the self-dual Yang-Mills equations to three dimensions," in Painleve Trascendents, Their Asymptotics and Physical Applications, D. Levi, Ed., pp. 393-405, Plenum Press, New York, 1992.

[28] K. Toda and S.-j. Yu, "The investigation into the SchwarzKorteweg-de Vries equation and the Schwarz derivative in (2 +1) dimensions," Journal of Mathematical Physics, vol. 41, no. 7, pp. 4747-4751, 2000.

[29] M. S. Bruzon, M. L. Gandarias, C. Muriel, J. Ramirez, S. Saez, and F. R. Romero, "The Calogero-Bogoyavlenskii-Schiff equation in 2+1 dimensions," Theoretical and Mathematical Physics, vol. 137, no. 1, pp. 1367-1377, 2003.

[30] B. G. Konopelchenko, Solitons in Multidimensions: Inverse Spectrum Transform Method, 1993.

[31] F. Calogero, "A method to generate solvable nonlinear evolution equations," Lettere Al Nuovo Cimento, vol. 14, no. 12, pp. 443-447, 1975.

[32] S.-J. Yu, K. Toda, N. Sasa, and T. Fukuyama, "Nsoliton solutions to the Bogoyavlenskii-Schiff equation and a quest for the soliton solution in (3 1) dimensions," Journal of Physics A: Mathematical and General, vol. 31, no. 14, pp. 3337-3347, 1998.

[33] K. Toda, S.-j. Yu, and T. Fukuyama, "The BogoyavlenskiiSchiff hierarchy and integrable equations in $(2+1)$ dimensions," Reports on Mathematical Physics, vol. 44, no. 1-2, pp. 247-254, 1999.

[34] A.-m. Wazwaz, "The (2+1) and (3+1)-dimensional CBS equations: multiple soliton solutions and multiple singular soliton solutions," Zeitschrift für Naturforschung A, vol. 65, no. 3, pp. 173-181, 2010.

[35] Taogetusang, "New infinite sequence soliton-like solutions of $(2+1)$-dimensional generalized Calogero-BogoyavlenskiiSchiff equation," Acta Physica Sinica, vol. 62, no. 21, p. 210201, 2013.

[36] B. Li and Y. Chen, "Exact analytical solutions of the generalized Calogero-Bogoyavlenskii-Schiff equation using symbolic computation," Czechoslovak journal of physics, vol. 54, no. 5, pp. 517-528, 2004.

[37] H. Yan, S. F. Tian, L. L. Feng, and T. T. Zhang, "Quasi-periodic wave solutions, soliton solutions, and integrability to a $(2+1)$ dimensional generalized Bogoyavlensky-Konopelchenko equation," Waves in Random and Complex Media, vol. 26, no. 4, pp. 444-457, 2016.

[38] H. Triki, Z. Jovanoski, and A. Biswas, "Shock wave solutions to the Bogoyavlensky-Konopelchenko equation," Indian Journal of Physics, vol. 88, no. 1, pp. 71-74, 2014.
[39] Y.-L. Wang, Y.-T. Gao, S.-L. Jia, G.-F. Deng, and H. WenQiang, "Solitons for a (2+1)-dimensional variable-coefficient Bogoyavlensky-Konopelchenko equation in a fluid," Modern Physics Letters B, vol. 31, no. 25, p. 1750216, 2017.

[40] J. Manafian, B. M. Ivatloo, and M. Abapour, "Breather wave, periodic, and cross-kink solutions to the generalized Bogoyavlensky-Konopelchenko equation," Mathematical Methods in the Applied Sciences, vol. 43, no. 4, pp. 17531774, 2019.

[41] Z. Dai, J. Liu, and D. Li, "Applications of HTA and EHTA to YTSF Equation," Applied Mathematics and Computation, vol. 207, no. 2, pp. 360-364, 2009.

[42] Z. Xu, H. Chen, and Z. Dai, "Rogue wave for the (2+1)-dimensional Kadomtsev-Petviashvili equation," Applied Mathematics Letters, vol. 37, pp. 34-38, 2014. 